\title{
A CONVERGENCE RESULT FOR MATRIX RICCATI DIFFERENTIAL EQUATIONS ASSOCIATED WITH $M$-MATRICES
}

\author{
CHUN-HUA GUO AND BO YU
}

\begin{abstract}
The initial value problem for a matrix Riccati differential equation associated with an $M$-matrix is known to have a global solution $X(t)$ on $[0, \infty)$ when $X(0)$ takes values from a suitable set of nonnegative matrices. It is also known, except for the critical case, that as $t$ goes to infinity $X(t)$ converges to the minimal nonnegative solution of the corresponding algebraic Riccati equation. In this paper we present a new approach for proving the convergence, which is based on the doubling procedure and is also valid for the critical case. The approach also provides a way for solving the initial value problem and a new doubling algorithm for computing the minimal nonnegative solution of the algebraic Riccati equation.
\end{abstract}

\section{INTRODUCTION}

We consider the initial value problem for the matrix Riccati differential equation (RDE):

$$
X^{\prime}(t)=X(t) C X(t)-X(t) D-A X(t)+B, \quad X(0)=X_{0},
$$

for which the block matrix

$$
K=\left(\begin{array}{cc}
D & -C \\
-B & A
\end{array}\right)
$$

is a nonsingular $M$-matrix or an irreducible singular $M$-matrix, where $A$ and $D$ are square matrices of orders $m$ and $n$, respectively. An excellent and quite comprehensive work on Riccati differential equations is the book [1].

For any real matrices $A=\left(a_{i j}\right)$ and $B=\left(b_{i j}\right)$ of the same size, $A \geq B(A>B)$ means $a_{i j} \geq b_{i j}\left(a_{i j}>b_{i j}\right)$ for all $i, j$. A real square matrix $A$ is called a $Z$-matrix if $a_{i j} \leq 0$ whenever $i \neq j$. A $Z$-matrix can be written as $s I-B$ with $B \geq 0$ and is called an $M$-matrix if $s \geq \rho(B)$, where $\rho(\cdot)$ denotes the spectral radius. It is called a nonsingular $M$-matrix if $s>\rho(B)$ and a singular $M$-matrix if $s=\rho(B)$. Let $\sigma(A)$ be the spectrum of a square matrix $A$, and let $\mathbb{C}_{<}, \mathbb{C}_{>}, \mathbb{C}_{\leq}$and $\mathbb{C}_{\geq}$be the open left half-plane, the open right half-plane, the closed left half-plane and the closed right half-plane, respectively. It is clear that $\sigma(A) \subset \mathbb{C}_{>}$for nonsingular $M$-matrices $A$ and $\sigma(A) \subset \mathbb{C}_{\geq}$for singular $M$-matrices $A$. A $Z$-matrix $A$ is a nonsingular $M$-matrix if and only if $A v>0$ for some positive vector $v$ (see [2] or $[10])$.

1991 Mathematics Subject Classification. 15A24, 34A12, 65F30.

Key words and phrases. Riccati differential equation, $M$-matrix, Global solution, Convergence, Doubling algorithm, Algebraic Riccati equation, Minimal nonnegative solution. 
If $K$ in (2) is an irreducible singular $M$-matrix, the Perron-Frobenius theorem [2] implies that there are positive vectors $\left(\begin{array}{c}u_{1} \\ u_{2}\end{array}\right)$ and $\left(\begin{array}{c}v_{1} \\ v_{2}\end{array}\right)$, where $u_{1}, v_{1} \in \mathbb{R}^{n}$ and $u_{2}, v_{2} \in \mathbb{R}^{m}$, such that

$$
K\left(\begin{array}{l}
v_{1} \\
v_{2}
\end{array}\right)=0, \quad\left(\begin{array}{ll}
T & u_{2}^{T}
\end{array}\right) K=0
$$

The study of the initial value problem (1) is closely related to that of the algebraic Riccati equation (ARE)

$$
X C X-X D-A X+B=0 .
$$

Nonsymmetric AREs of this type arise in transport theory (see [13]) and WienerHopf factorization of Markov chains (see [14]). The solution of practical interest is the minimal nonnegative solution.

The following result is obtained by combining various results from [6] and [7] (see [6, Theorem 3.1, Theorem 4.2, Theorem 4.7] and [7, Theorem 5]).

Theorem 1. The equation (4) has a minimal nonnegative solution $\Phi$ (the minimality is defined using the elementwise order for matrices) and its dual equation

$$
Y B Y-Y A-D Y+C=0
$$

(obtained from (4) by switching $A$ and $D$ and switching $B$ and $C$ ) has a minimal nonnegative solution $\Psi$. Moreover, $\Phi, \Psi>0$ when $K$ is irreducible. If $K$ in $(2)$ is a nonsingular $M$-matrix, then $D-C \Phi$ and $A-B \Psi$ are nonsingular $M$-matrices. If $K$ is an irreducible singular $M$-matrix with $u_{1}^{T} v_{1} \neq u_{2}^{T} v_{2}$, then one of $D-C \Phi$ and $A-B \Psi$ is an irreducible nonsingular $M$-matrix and the other is an irreducible singular $M$-matrix. If $K$ is an irreducible singular $M$-matrix with $u_{1}^{T} v_{1}=u_{2}^{T} v_{2}$, then both $D-C \Phi$ and $A-B \Psi$ are irreducible singular $M$-matrices.

It is known [6] that the matrix

$$
P=\left(\begin{array}{cc}
I & \Psi \\
\Phi & I
\end{array}\right)
$$

is nonsingular (or equivalently $\rho(\Phi \Psi)<1$ ) if $K$ in $(2)$ is a nonsingular $M$-matrix or an irreducible singular $M$-matrix with $u_{1}^{T} v_{1} \neq u_{2}^{T} v_{2}$ and that $P$ is singular if $K$ is an irreducible singular $M$-matrix with $u_{1}^{T} v_{1}=u_{2}^{T} v_{2}$, which will be referred to as the critical case.

By Theorem 6 of [5], the initial value problem (1) has a global solution $X(t)$ on $[0, \infty)$ if $0 \leq X_{0} \leq \widetilde{\Phi}$, where $\widetilde{\Phi}$ is any nonnegative solution of (4). Two results about the convergence of $X(t)$ to $\Phi$ are proved in [5] (see Theorems 9 and 13 there). However, the proof in [5] is not valid for the critical case since it requires the nonsingularity of the matrix $P$ in (6).

In this paper we present a new approach for proving the convergence of $X(t)$ to $\Phi$, which is based on the doubling procedure. The new approach will establish the convergence in the critical case as well. For a special RDE corresponding to the ARE arising in transport theory (see [11] and [13]), a convergence result in the critical case has been proved in [12], where the assumption on $X_{0}$ is also more restrictive (see [12, Theorem 4.2]). For symmetric RDEs, convergence results for the solutions of initial value problems have been given in $[3,15,16]$. 
The new approach in this paper also provides a way for solving the initial value problem and a new doubling algorithm for computing the minimal nonnegative solution of the algebraic Riccati equation.

\section{Convergence proof Based on a doubling procedure}

Let $\Phi$ and $\Psi$ be the minimal nonnegative solutions of the ARE (4) and the dual ARE (5), respectively. Let

$$
H=\left(\begin{array}{ll}
D & -C \\
B & -A
\end{array}\right) .
$$

Then it is easily verified (and is well known) that

$$
H\left(\begin{array}{cc}
I & \Psi \\
\Phi & I
\end{array}\right)=\left(\begin{array}{cc}
I & \Psi \\
\Phi & I
\end{array}\right)\left(\begin{array}{cc}
R & 0 \\
0 & -S
\end{array}\right)
$$

where $R=D-C \Phi$ and $S=A-B \Psi$.

The eigenvalues of $H$ are the collection of the eigenvalues of $R$ and $-S$. The fact is not obvious for the critical case where the matrix $P$ in (6) is singular, but is proved in [6]. Moreover, $H$ has a double eigenvalue at 0 with a $2 \times 2$ Jordan block in the critical case [6].

Suppose that $0 \leq X_{0} \leq \widetilde{\Phi}$, where $\widetilde{\Phi}$ is any nonnegative solution of (4). Then, by Theorem 6 of [5], the initial value problem (1) has a solution $X(t)$ on $[0, \infty)$. We also have (see [5, Lemma 7] for example) that $X(t)=Z(t) Y^{-1}(t)$, where

$$
\left(\begin{array}{c}
Y(t) \\
Z(t)
\end{array}\right)=e^{H t}\left(\begin{array}{c}
I \\
X_{0}
\end{array}\right) .
$$

Since we would like to present a proof of convergence for the critical case, the relation in (8) does not give a similarilty transformation for $H$. So we will use a new approach in this paper.

The pencil $\lambda I-e^{H t}$ is equivalent to the pencil $\lambda M(t)-L(t)$ whenever $M(t)$ is nonsingular and $M(t)^{-1} L(t)=e^{H t}$. We now require that the matrices $L(t), M(t)$ have the following structures.

$$
L(t)=\left(\begin{array}{cc}
I & -G(t) \\
0 & F(t)
\end{array}\right), \quad M(t)=\left(\begin{array}{cc}
E(t) & 0 \\
-H(t) & I
\end{array}\right)
$$

Note that the structures in (10) are as in [4] and [9]. But here the matrices $E(t), F(t), G(t), H(t)$ are to be determined through the relation $M(t)^{-1} L(t)=e^{H t}$. Let

$$
e^{H t}=\left(\begin{array}{ll}
\Gamma_{11}(t) & \Gamma_{12}(t) \\
\Gamma_{21}(t) & \Gamma_{22}(t)
\end{array}\right),
$$

where the partitioning is the same as in (7). It follows from $M(t)^{-1} L(t)=e^{H t}$ that

$$
\begin{aligned}
E(t) & =\Gamma_{11}^{-1}, \\
F(t) & =\Gamma_{22}-\Gamma_{21} \Gamma_{11}^{-1} \Gamma_{12}, \\
G(t) & =-\Gamma_{11}^{-1} \Gamma_{12}, \\
H(t) & =\Gamma_{21} \Gamma_{11}^{-1},
\end{aligned}
$$


provided $\Gamma_{11}$ is nonsingular, where we have omitted the variable $t$ in all $\Gamma_{i j}$. For $t>0$ sufficiently small, $\Gamma_{11}(t)$ is close to the identity matrix and is thus nonsingular. It will be shown later in the paper that $\Gamma_{11}(t)$ is actually nonsingular for all $t>0$.

We now express $X(t)$ in terms of $E(t), F(t), G(t), H(t)$, assuming that $E(t)$ (or equivalently $\left.\Gamma_{11}(t)\right)$ is nonsingular. Using $e^{H t}=M(t)^{-1} L(t)$ in (9) yields

$$
Y(t)=E(t)^{-1}\left(I-G(t) X_{0}\right), \quad Z(t)=F(t) X_{0}+H(t) Y(t) .
$$

It follows that

$$
X(t)=Z(t) Y(t)^{-1}=H(t)+F(t) X_{0}\left(I-G(t) X_{0}\right)^{-1} E(t) .
$$

Our convergence proof for $X(t)$ will be based a doubling procedure, which we now describe.

By (8) and the power series expansion of the exponential function, we have

$$
e^{H t}\left(\begin{array}{cc}
I & \Psi \\
\Phi & I
\end{array}\right)=\left(\begin{array}{cc}
I & \Psi \\
\Phi & I
\end{array}\right)\left(\begin{array}{cc}
e^{R t} & 0 \\
0 & e^{-S t}
\end{array}\right)
$$

which is equivalent to

$$
\begin{aligned}
& L(t)\left(\begin{array}{l}
I \\
\Phi
\end{array}\right) e^{-R t}=M(t)\left(\begin{array}{l}
I \\
\Phi
\end{array}\right), \\
& L(t)\left(\begin{array}{l}
\Psi \\
I
\end{array}\right)=M(t)\left(\begin{array}{l}
\Psi \\
I
\end{array}\right) e^{-S t} .
\end{aligned}
$$

We now take $\eta>0$ such that $\Gamma_{11}(\eta)$ is nonsingular, and let

$$
E_{0}=E(\eta), F_{0}=F(\eta), G_{0}=G(\eta), H_{0}=H(\eta) .
$$

So for $L_{0}=L(\eta), M_{0}=M(\eta)$ we have

$$
\begin{aligned}
& L_{0}\left(\begin{array}{l}
I \\
\Phi
\end{array}\right) e^{-R \eta}=M_{0}\left(\begin{array}{l}
I \\
\Phi
\end{array}\right) \\
& L_{0}\left(\begin{array}{l}
\Psi \\
I
\end{array}\right)=M_{0}\left(\begin{array}{l}
\Psi \\
I
\end{array}\right) e^{-S \eta} .
\end{aligned}
$$

We then generate

$$
L_{k}=\left(\begin{array}{cc}
I & -G_{k} \\
0 & F_{k}
\end{array}\right), \quad M_{k}=\left(\begin{array}{cc}
E_{k} & 0 \\
-H_{k} & I
\end{array}\right)
$$

by the following doubling algorithm, assuming no breakdown occurs.

Algorithm 2. Let $E_{0}, F_{0}, G_{0}, H_{0}$ be given by (20). Generate the sequences $\left\{E_{k}\right\}$, $\left\{F_{k}\right\},\left\{G_{k}\right\},\left\{H_{k}\right\}$ using the doubling procedure

$$
\begin{aligned}
E_{k+1} & =E_{k}\left(I-G_{k} H_{k}\right)^{-1} E_{k}, \\
F_{k+1} & =F_{k}\left(I-H_{k} G_{k}\right)^{-1} F_{k}, \\
G_{k+1} & =G_{k}+E_{k}\left(I-G_{k} H_{k}\right)^{-1} G_{k} F_{k}, \\
H_{k+1} & =H_{k}+F_{k}\left(I-H_{k} G_{k}\right)^{-1} H_{k} E_{k} .
\end{aligned}
$$

We remark that the doubling procedure in (24)-(27) is exactly the same as in $[4,9]$. But the initialization in the algorithm is now given by $(20)$, unlike that in $[4,9]$. We will show later (in Theorem 8) that no breakdown occurs in Algorithm 2. By the property of the doubling procedure (see $[4,9]$ ), we have

$$
M_{k}^{-1} L_{k}=\left(M_{0}^{-1} L_{0}\right)^{2^{k}}=\left(e^{H \eta}\right)^{2^{k}}=e^{H 2^{k} \eta} .
$$


This implies that $\Gamma_{11}\left(2^{k} \eta\right)$ is nonsingular. So $L_{k}$ and $M_{k}$ are uniquely determined by $e^{H 2^{k} \eta}$. We then have

$$
L_{k}=L\left(2^{k} \eta\right), \quad M_{k}=M\left(2^{k} \eta\right)
$$

It follows from (16) that $X\left(2^{k} \eta\right)$ is determined by $L_{k}$ and $M_{k}$. We also have (as in $[4,9])$ that

$$
\begin{aligned}
& L_{k}\left(\begin{array}{c}
I \\
\Phi
\end{array}\right)\left(e^{-R \eta}\right)^{2^{k}}=M_{k}\left(\begin{array}{c}
I \\
\Phi
\end{array}\right), \\
& L_{k}\left(\begin{array}{l}
\Psi \\
I
\end{array}\right)=M_{k}\left(\begin{array}{c}
\Psi \\
I
\end{array}\right)\left(e^{-S \eta}\right)^{2^{k}} .
\end{aligned}
$$

To show the convergence of $X(t)$, we need to show the convergence of $E_{k}, F_{k}, G_{k}, H_{k}$ for $\eta>0$ sufficiently small. We start with proving that Algorithm 2 is well defined for all $\eta>0$. So $\Gamma_{11}(t)$ in (11) is actually nonsingular for all $t>0$.

Lemma 3. Suppose that the matrix $K$ in (2) has no zero entries and $E_{0}, F_{0}, G_{0}, H_{0}$ are given by (20). Then there exists $\eta^{*}>0$ such that $E_{0}, F_{0}, G_{0}, H_{0}>0$ and $I-G_{0} H_{0}$ and $I-H_{0} G_{0}$ are nonsingular $M$-matrices when $0<\eta \leq \eta^{*}$.

Proof. We have

$$
e^{H t}=I+\left(\begin{array}{ll}
D & -C \\
B & -A
\end{array}\right) t+O\left(t^{2}\right) .
$$

Then $\Gamma_{11}(t)=I+D t+O\left(t^{2}\right)$. So $\Gamma_{11}$ is nonsingular for all $t$ sufficiently small.

Let $D=s_{1} I-N_{1}$ with $s_{1}>0$ and $N_{1}>0$. Then we have

$$
\begin{aligned}
E(t) & =\Gamma_{11}^{-1}=\frac{1}{1+s_{1} t}\left(I-\frac{t}{1+s_{1} t} N_{1}+O\left(t^{2}\right)\right)^{-1} \\
& =\frac{1}{1+s_{1} t} I+\frac{t}{\left(1+s_{1} t\right)^{2}} N_{1}+O\left(t^{2}\right) \\
& >0
\end{aligned}
$$

for all $t \in\left(0, t_{1}\right]$, for some $t_{1}>0$ sufficiently small. As $B$ and $C$ are positive matrices,

$$
G(t)=-\Gamma_{11}^{-1} \Gamma_{12}=E(t)\left(C t+O\left(t^{2}\right)\right)>0
$$

for all $t \in\left(0, t_{2}\right]$ and

$$
H(t)=\Gamma_{21} \Gamma_{11}^{-1}=\left(B t+O\left(t^{2}\right)\right) E(t)>0
$$

for all $t \in\left(0, t_{3}\right]$. Let $A=s_{2} I-N_{2}$ with $s_{2}>0$ and $N_{2}>0$. Then

$$
\begin{aligned}
F(t) & =\Gamma_{22}-\Gamma_{21} \Gamma_{11}^{-1} \Gamma_{12} \\
& =I-A t+O\left(t^{2}\right)-\Gamma_{21} \Gamma_{11}^{-1} \Gamma_{12} \\
& =\left(1-s_{2} t\right) I+N_{2} t-\Gamma_{21} \Gamma_{11}^{-1} \Gamma_{12}+O\left(t^{2}\right) \\
& >0
\end{aligned}
$$

for all $t \in\left(0, t_{4}\right]$.

Since $\lim _{t \rightarrow 0} e^{H t}=I$, we have $\lim _{t \rightarrow 0} G(t) H(t)=0$. So

$$
\rho(G(t) H(t))=\rho(H(t) G(t))<1 \text { for all } t \in\left(0, t_{5}\right] .
$$

Now the conclusions in the theorem hold with $\eta^{*}=\min \left\{t_{1}, t_{2}, t_{3}, t_{4}, t_{5}\right\}$. 
We will need to get rid of the assumption that the matrix $K$ in (2) has no zero entries. A direct use of continuity argument would not work since the number $\eta^{*}$ in Lemma 3 may decrease to 0 in a limit process. We will then achieve our goal in a roundabout way. The following result about matrix exponential can be found in [17, Section 8.2] for example.

Lemma 4. For any $Z$-matrix $A, e^{-A t} \geq 0$ when $t \geq 0$; for any irreducible $Z$-matrix $A, e^{-A t}>0$ when $t>0$.

With this lemma, we can obtain the following result about Algorithm 2.

Lemma 5. Let $\Phi$ and $\Psi$ be the minimal nonnegative solutions of the ARE (4) and the dual ARE (5), respectively. Suppose that $K$ has no zero entries and $E_{0}, F_{0}, G_{0}, H_{0}$ are given by (20) with $0<\eta \leq \eta^{*}$, where $\eta^{*}$ is as in Lemma 3. Then the sequences $\left\{E_{k}\right\},\left\{F_{k}\right\},\left\{G_{k}\right\},\left\{H_{k}\right\}$ in Algorithm 2 are well defined. Moreover, for all $k \geq 0, E_{k}>0, F_{k}>0,0<H_{k}<H_{k+1}<\Phi, 0<G_{k}<G_{k+1}<\Psi$, and both $I-G_{k} H_{k}$ and $I-H_{k} G_{k}$ are nonsingular $M$-matrices.

Proof. The proof can be completed by induction as in [9].

The next result says that the conclusions in the above lemma actually hold for all $\eta>0$.

Lemma 6. Suppose that $K$ has no zero entries and $E_{0}, F_{0}, G_{0}, H_{0}$ are given by (20) with any $\eta>0$. Then the sequences $\left\{E_{k}\right\},\left\{F_{k}\right\},\left\{G_{k}\right\},\left\{H_{k}\right\}$ in Algorithm 2 are well defined, $E_{k}>0, F_{k}>0,0<H_{k}<H_{k+1}<\Phi, 0<G_{k}<G_{k+1}<\Psi$, and both $I-G_{k} H_{k}$ and $I-H_{k} G_{k}$ are nonsingular $M$-matrices.

Proof. We take $k_{0}$ large enough so that $0<\eta / 2^{k_{0}} \leq \eta^{*}$. Let $\widehat{E}_{0}=E\left(\eta / 2^{k_{0}}\right), \widehat{F}_{0}=$ $F\left(\eta / 2^{k_{0}}\right), \widehat{G}_{0}=G\left(\eta / 2^{k_{0}}\right), \widehat{H}_{0}=H\left(\eta / 2^{k_{0}}\right)$. By Lemma 5 , the sequences $\left\{\widehat{E}_{k}\right\},\left\{\widehat{F}_{k}\right\}$, $\left\{\widehat{G}_{k}\right\},\left\{\widehat{H}_{k}\right\}$ are well defined by Algorithm $2, \widehat{E}_{k}>0, \widehat{F}_{k}>0,0<\widehat{H}_{k}<\widehat{H}_{k+1}<\Phi$, $0<\widehat{G}_{k}<\widehat{G}_{k+1}<\Psi$, and both $I-\widehat{G}_{k} \widehat{H}_{k}$ and $I-\widehat{H}_{k} \widehat{G}_{k}$ are nonsingular $M$ matrices. We only need to show that $E_{0}=\widehat{E}_{k_{0}}, F_{0}=\widehat{F}_{k_{0}}, G_{0}=\widehat{G}_{k_{0}}, H_{0}=\widehat{H}_{k_{0}}$. In fact, by the property of the doubling procedure, we now have

$$
\widehat{M}_{k_{0}}^{-1} \widehat{L}_{k_{0}}=\left(\widehat{M}_{0}^{-1} \widehat{L}_{0}\right)^{2^{k_{0}}}=\left(e^{H \eta / 2^{k_{0}}}\right)^{2^{k_{0}}}=e^{H \eta},
$$

where $\widehat{L}_{k}$ and $\widehat{M}_{k}$ are defined by $\widehat{E}_{k}, \widehat{F}_{k}, \widehat{G}_{k}, \widehat{H}_{k}$ as in (23). From this we have $I=$ $\widehat{E}_{k_{0}} \Gamma_{11}(\eta)$. So $\Gamma_{11}(\eta)$ is nonsingular and $L(\eta)$ and $M(\eta)$ are uniquely determined by $M(\eta)^{-1} L(\eta)=e^{H \eta}$. Thus

$$
L(\eta)=\widehat{L}_{k_{0}}, \quad M(\eta)=\widehat{M}_{k_{0}} .
$$

Equivalently, $E_{0}=\widehat{E}_{k_{0}}, F_{0}=\widehat{F}_{k_{0}}, G_{0}=\widehat{G}_{k_{0}}, H_{0}=\widehat{H}_{k_{0}}$.

We can now drop the assumption that $K$ has no zero entries.

Theorem 7. Let $E_{0}, F_{0}, G_{0}, H_{0}$ be given by (20) for any $\eta>0$. Then $E_{0}, F_{0}, G_{0}$, $H_{0} \geq 0$ and $I-G_{0} H_{0}$ and $I-H_{0} G_{0}$ are nonsingular $M$-matrices.

Proof. Since $K$ is a nonsingular $M$-matrix or an irreducible singular $M$-matrix, there is vector $v>0$ be such that $K v \geq 0$. Let $K_{\epsilon}=K+2 \epsilon e^{T} v I-\epsilon v e^{T}$ with $\epsilon>0$, where $e$ is the vector of ones. Then $K_{\epsilon} v=K v+\epsilon\left(e^{T} v\right) v>0$. So $K_{\epsilon}$ is a nonsingular $M$-matrix with no zero entries. We can now determine

$$
L_{\epsilon}(t)=\left(\begin{array}{cc}
I & -G_{\epsilon}(t) \\
0 & F_{\epsilon}(t)
\end{array}\right), \quad M_{\epsilon}(t)=\left(\begin{array}{cc}
E_{\epsilon}(t) & 0 \\
-H_{\epsilon}(t) & I
\end{array}\right)
$$


by $M_{\epsilon}(t)^{-1} L_{\epsilon}(t)=e^{H_{\epsilon} t}$ for any $t>0$, where $H_{\epsilon}=\left(\begin{array}{cc}I_{n} & 0 \\ 0 & -I_{m}\end{array}\right) K_{\epsilon}$.

Since $\lim _{t \rightarrow 0} e^{H t}=I$, we can find an $\eta>0$ such that $E(\eta)$ is nonsingular and $\rho(G(\eta) H(\eta))=\rho(H(\eta) G(\eta))<1$. By Lemma 6

$$
E_{\epsilon}(\eta), F_{\epsilon}(\eta), G_{\epsilon}(\eta), H_{\epsilon}(\eta)>0
$$

for this $\eta$ and all $\epsilon>0$. Letting $\epsilon \rightarrow 0$ yields

$$
E(\eta), F(\eta), G(\eta), H(\eta) \geq 0 .
$$

So the conclusions in the theorem hold for this fixed $\eta$. Then the arguments in the proof of Lemma 6 show that the conclusions also hold for any $\eta>0$.

We then have the following convergence result for Algorithm 2.

Theorem 8. Let $E_{0}, F_{0}, G_{0}, H_{0}$ be given by (20) with any $\eta>0$. Then the sequences $\left\{E_{k}\right\},\left\{F_{k}\right\},\left\{G_{k}\right\},\left\{H_{k}\right\}$ in Algorithm 2 are well defined, $E_{k} \geq 0, F_{k} \geq 0$, $0 \leq H_{k} \leq H_{k+1} \leq \Phi, 0 \leq G_{k} \leq G_{k+1} \leq \Psi$, and both $I-G_{k} H_{k}$ and $I-H_{k} G_{k}$ are nonsingular $M$-matrices. Moreover, we have

$$
\begin{aligned}
& \limsup \sqrt[2^{k}]{\left\|\Phi-H_{k}\right\|} \leq \rho\left(e^{-R \eta}\right) \rho\left(e^{-S \eta}\right), \\
& \limsup \sqrt[2^{k}]{\left\|\Psi-G_{k}\right\|} \leq \rho\left(e^{-R \eta}\right) \rho\left(e^{-S \eta}\right), \\
& \limsup \sqrt[2^{k}]{\left\|E_{k}\right\|} \leq \rho\left(e^{-R \eta}\right) \\
& \limsup \sqrt[2^{k}]{\left\|F_{k}\right\|} \leq \rho\left(e^{-S \eta}\right) .
\end{aligned}
$$

The sequence $\left\{E_{k}\right\}$ is still bounded when $\rho\left(e^{-R \eta}\right)=1$. The sequence $\left\{F_{k}\right\}$ is still bounded when $\rho\left(e^{-S \eta}\right)=1$.

Proof. With Lemma 4, the proof can be completed by induction as in [9] and [8]. Note that we do not require $E_{0} e, F_{0} e>0$ when using the arguments in [8] to show that $I-G_{k} H_{k}$ and $I-H_{k} G_{k}$ are nonsingular $M$-matrices.

When $K$ is a nonsingular $M$-matrix, both $R$ and $S$ are nonsingular $M$-matrices. When $K$ is an irreducible singular $M$-matrix, for the critical case both $R$ and $S$ are irreducible singular $M$-matrices and for the non-critical case one of $R$ and $S$ is an irreducible singular $M$-matrix and the other is an irreducible nonsingular $M$ matrix. From the eigenvalue location of $M$-matrices we know that, for any $t>0$, $\rho\left(e^{-Q t}\right)<1$ if $Q$ is a nonsingular $M$-matrix and $\rho\left(e^{-Q t}\right)=1$ if $Q$ is a singular $M$-matrix. Theorem 8 then shows that, except for the critical case, $H_{k}$ converges to $\Phi$ quadratically and $G_{k}$ converges to $\Psi$ quadratically.

We now examine the critical case.

Theorem 9. Let $E_{0}, F_{0}, G_{0}, H_{0}$ be given by (20) with any $\eta>0$ and the sequences $\left\{E_{k}\right\},\left\{F_{k}\right\},\left\{G_{k}\right\},\left\{H_{k}\right\}$ be generated by Algorithm 2. In the critical case we have

$$
E_{k} \rightarrow 0, \quad F_{k} \rightarrow 0, \quad H_{k} \rightarrow \Phi, \quad G_{k} \rightarrow \Psi,
$$

and the convergence of each sequence is at least linear with rate $1 / 2$.

Proof. The proof is largely the same as the proof of [4, Theorem 5.4]. The difference is only in the beginning.

For the critical case, the matrix $H$ (and thus $H \eta$ ) has $n-1$ eigenvalues in $\mathbb{C}_{>}$, $m-1$ eigenvalues in $\mathbb{C}_{<}$, and two eigenvalues at 0 with a $2 \times 2$ Jordan block. Thus, the matrix $e^{H \eta}$ has $n-1$ eigenvalues outside the unit circle, $m-1$ eigenvalues inside 
the unit circle, and two eigenvalues at 1 with a $2 \times 2$ Jordan block. Here we have used the fact that the exponential of the matrix $\left(\begin{array}{ll}0 & 1 \\ 0 & 0\end{array}\right)$ is the matrix $\left(\begin{array}{ll}1 & 1 \\ 0 & 1\end{array}\right)$.

Now for suitable nonsingular matrices $V$ and $Z$ we have

$$
\begin{aligned}
V L_{0} Z & =\left(\begin{array}{cc}
I_{n} & 0_{n, m} \\
0_{m, n} & J_{2, s} \oplus(1)
\end{array}\right), \\
V M_{0} Z & =\left(\begin{array}{cc}
J_{1, s} \oplus(1) & 0_{n-1, m-1} \oplus(1) \\
0_{m, n} & I_{m-1} \oplus(1)
\end{array}\right),
\end{aligned}
$$

where $X \oplus Y$ means $\left(\begin{array}{cc}X & 0 \\ 0 & Y\end{array}\right)$, and for suitable nonsingular matrices $T$ and $W$ we have

$$
\begin{aligned}
T L_{0} W & =\left(\begin{array}{cc}
J_{2, s} \oplus(1) & 0_{m-1, n-1} \oplus(1) \\
0_{n, m} & I_{n-1} \oplus(1)
\end{array}\right), \\
T M_{0} W & =\left(\begin{array}{cc}
I_{m} & 0_{m, n} \\
0_{n, m} & J_{1, s} \oplus(1)
\end{array}\right),
\end{aligned}
$$

where $J_{1, s} \oplus(1)$ is similar to $e^{-R \eta}$ and $J_{2, s} \oplus(1)$ is similar to $e^{-S \eta}$, with $\rho\left(J_{1, s}\right)<1$ and $\rho\left(J_{2, s}\right)<1$. The rest of the proof is the same as in [4]. (1).

We now return to the convergence of $X(t)$, the solution to the intial value problem

When $K$ is irreducible, it is shown in [5] that the ARE (4) has a positive solution $\Phi_{+}$with $\rho\left(\Psi \Phi_{+}\right)=1$ and that $\Phi_{+}>\Phi$ for the non-critical case and $\Phi_{+}=\Phi$ in the critical case.

The following result includes [5, Theorem 9] and [5, Theorem 13] as special cases. What is new here is the convergence of $X(t)$ in the critical case. Our unified proof is based on the convergence results for Algorithm 2, obtained earlier in this paper.

Theorem 10. If $0 \leq X_{0} \leq \Phi$, then $X(t) \rightarrow \Phi$ as $t \rightarrow \infty$. If $K$ is ireducible, $0 \leq X_{0} \leq \Phi_{+}$and $X_{0} \neq \Phi_{+}$, then $X(t) \rightarrow \Phi$ as $t \rightarrow \infty$.

Proof. If $X_{0}=\Phi$, then we have $X(t)=\Phi$ for all $t \geq 0$. We now assume that $X_{0} \neq \Phi$. If $K$ is a nonsingular $M$-matrix and $0 \leq X_{0} \leq \Phi$, it follows from Perron-Frobenius theory [2] that $\rho\left(\Psi X_{0}\right) \leq \rho(\Psi \Phi)<1$. If $K$ is an irreducible $M$-matrix, $0 \leq X_{0} \leq \Phi_{+}$and $X_{0} \neq \Phi_{+}$, it follows from Perron-Frobenius theory that $\rho\left(\Psi X_{0}\right)<\rho\left(\Psi \Phi_{+}\right)=1$. Thus $I-\Psi X_{0}$ is a nonsingular $M$-matrix in either case. Now for any $\eta>0$ we have by (16) and (28) that

$$
X\left(2^{k} \eta\right)=H_{k}+F_{k} X_{0}\left(I-G_{k} X_{0}\right)^{-1} E_{k},
$$

where the sequences $\left\{E_{k}\right\},\left\{F_{k}\right\},\left\{G_{k}\right\},\left\{H_{k}\right\}$ are generated by Algorithm 2, with $E_{0}, F_{0}, G_{0}, H_{0}$ be given by $(20)$. By Theorems 8 and 9 , we know that $X\left(2^{k} \eta\right)$ converges to $\Phi$ as $k \rightarrow \infty$, noting that $\lim _{k \rightarrow \infty} X_{0}\left(I-G_{k} X_{0}\right)^{-1}=X_{0}\left(I-\Psi X_{0}\right)^{-1}$ exits. Since $\eta>0$ is arbitrary, it follows that $X(t) \rightarrow \Phi$ as $t \rightarrow \infty$.

\section{Discussions}

The formula (45) also provides a way for solving the initial value problem (1), assuming that $X_{0}$ is in the range described in Theorem 10. Without this assumption, (1) may not have a global solution on $[0, \infty)$. When using (45) to approximate $X(t)$, the approximations are computed at mesh points of the form $2^{k} \eta$, where 
$\eta>0$ is a small number. So the mesh grid here is not uniform at all; the grid is fine near the origin and coarse away from the origin. This seems to be reasonable since the variations of $X(t)$ become small for large $t$ values. The formula (9) cannot be used directly for the computation of $X(t)$ for large $t$ since overflow will occur in the computation of $e^{H t}$.

Theorems 8 and 9 show that the minimal solutions $\Phi$ and $\Psi$ can be approximated by Algorithm 2, where the initialization is given by (20). The doubling algorithm here is different from the doubling algorithms in [9] and [18], where the Cayley transform and the generalized Cayley transform are used, respectively. In general, the doubling algorithms in [9] and [18] are less expensive than Algorithm 2, which requires the computation of matrix exponential in the initialization, but they do not solve the initial value problem (1) at the same time. If the purpose is just to compute the minimal solutions $\Phi$ and $\Psi$, Algorithm 2 may still be more efficient than the doubling algorithms in $[9,18]$, but only in some special situations. The number of iterations required for Algorithm 2 may be smaller than that required for the doubling algorithms in $[9,18]$ if $\eta>0$ is not too small. In theory, we can have very fast convergence for Algorithm 2 by taking $\eta$ to be large. In practice, however, when $\eta$ is large the matrix $\Gamma_{11}(\eta)$ is often ill-conditioned and the initial matrices $E_{0}, F_{0}, G_{0}, H_{0}$ in Algorithm 2 cannot be obtained accurately. The computation of matrix exponential in the initialization of Algorithm 2 is significantly more expensive than the initializations based on (generalized) Cayley transform. But if we need to compute the minimal solution $\Phi$ for the ARE (4) involving a parameter, it is possible to reduce the computational work required for computing the matrix exponential corresponding to one value of the parameter using the matrix exponential already computed for a nearby value of the parameter. For example, if $H=H_{1}+\epsilon H_{2}$ with $H_{1} H_{2}=H_{2} H_{1}$ and $e^{H_{1} \eta}$ is already computed, then we can use $e^{H \eta}=e^{H_{1} \eta} e^{\epsilon H_{2} \eta}$ and a lower order Taylor polynomial approximating $e^{\epsilon H_{2} \eta}$ to get a good approximation for $e^{H \eta}$.

\section{ACKNOWLEDGMents}

The work of C.G. was supported in part by a grant from the Natural Sciences and Engineering Research Council of Canada. The work of B.Y. was supported in part by NSF of China grant 11301170 .

\section{REFERENCES}

[1] H. Abou-Kandil, G. Freiling, V. Ionescu and G. Jank, Matrix Riccati Equations in Control and Systems Theory, Birkhäuser Verlag, Basel, 2003.

[2] A. Berman and R. J. Plemmons, Nonnegative Matrices in the Mathematical Sciences, Academic Press, New York, 1979.

[3] F. M. Callier and J. Winkin, Convergence of the time-invariant Riccati differential equation towards its strong solution for stabilizable systems, J. Math. Anal. Appl., 192 (1995), 230257.

[4] C.-Y. Chiang, E. K.-W. Chu, C.-H. Guo, T.-M. Huang, W.-W. Lin and S.-F. Xu, Convergence analysis of the doubling algorithm for several nonlinear matrix equations in the critical case, SIAM J. Matrix Anal. Appl., 31 (2009), 227-247.

[5] S. Fital and C.-H. Guo, Convergence of the solution of a nonsymmetric matrix Riccati differential equation to its stable equilibrium solution, J. Math. Anal. Appl., 318 (2006), 648-657.

[6] C.-H. Guo, Nonsymmetric algebraic Riccati equations and Wiener-Hopf factorization for M-matrices, SIAM J. Matrix Anal. Appl., 23 (2001), 225-242.

[7] C.-H. Guo, A note on the minimal nonnegative solution of a nonsymmetric algebraic Riccati equation, Linear Algebra Appl., 357 (2002), 299-302. 
[8] C.-H. Guo, B. Iannazzo and B. Meini, On the doubling algorithm for a (shifted) nonsymmetric algebraic Riccati equation, SIAM J. Matrix Anal. Appl., 29 (2007), 1083-1100.

[9] X.-X. Guo, W.-W. Lin and S.-F. Xu, A structure-preserving doubling algorithm for nonsymmetric algebraic Riccati equation, Numer. Math., 103 (2006), 393-412.

[10] R. A. Horn and C. R. Johnson, Topics in Matrix Analysis, Cambridge University Press, Cambridge, 1991.

[11] J. Juang, Existence of algebraic matrix Riccati equations arising in transport theory, Linear Algebra Appl., 230 (1995), 89-100.

[12] J. Juang, Global existence and stability of solutions of matrix Riccati equations, J. Math. Anal. Appl., 258 (2001), 1-12.

[13] J. Juang and W.-W. Lin, Nonsymmetric algebraic Riccati equations and Hamiltonian-like matrices, SIAM J. Matrix Anal. Appl., 20 (1998), 228-243.

[14] L. C. G. Rogers, Fluid models in queueing theory and Wiener-Hopf factorization of Markov chains, Ann. Appl. Probab., 4 (1994), 390-413.

[15] L. A. Safonov and V. V. Strygin, The convergence of the solution of a matrix Riccati equation to the maximal stationary solution in the critical case, Differential Equations, 38 (2002), 2934.

[16] M. A. Shayman, Phase portrait of the matrix Riccati equation, SIAM J. Control Optim., 24 (1986), 1-65.

[17] R. S. Varga, Matrix Iterative Analysis, 2nd ed., Springer, Berlin, 2000.

[18] W.-G. Wang, W.-C. Wang and R.-C. Li, Alternating-directional doubling algorithm for $M$ matrix algebraic Riccati equations, SIAM J. Matrix Anal. Appl., 33 (2012), 170-194.

Department of Mathematics and Statistics, University of Regina, Regina, SK S4S 0A2, CANADA

E-mail address: chun-hua.guo@uregina.ca

School of Science, Hunan University of Technology, Zhuzhou, Hunan 412000, P. R. CHINA

E-mail address: boyu_hut@126.com 